\title{
Correction to: Evaluation of treatment response in hepatocellular carcinoma in the explanted liver with Liver Imaging Reporting and Data System version 2017
}

\author{
Nieun Seo ${ }^{1}$ Myoung Soo Kim ${ }^{2} \cdot$ Mi-Suk Park ${ }^{1}$ (D) Jin-Young Choi ${ }^{1} \cdot$ Richard K. G. Do $^{3} \cdot$ Kyunghwa Han $^{4}$. \\ Myeong-Jin Kim ${ }^{1}$
}

Published online: 23 November 2020

(C) European Society of Radiology 2020

\section{Correction to: European Radiology (2020) 30:261-271 https://doi.org/10.1007/s00330-019-06376-5}

The original version of this article, published on 15 August 2019, unfortunately contained a mistake. The following correction has therefore been made in the original: The presentation of Table 3 was incorrect, the corrected table is given below. In addition, the incorrect values were cited in the third sentence of paragraph "Comparison of overall diagnostic performance to assess tumor viability: LI-RADS treatment response algorithm versus mRECIST." The sentence should read: "Both readers assigned equivocal category more frequently on CT rather than on MRI ( $10.3 \%$ vs. $6.0 \%$ in reader 1 , and $8.4 \%$ vs. $4.8 \%$ in reader 2, respectively; Fig. 3)." The original article has been corrected.

The online version of the original article can be found at https://doi.org/ 10.1007/s00330-019-06376-5

Mi-Suk Park

radpms@yuhs.ac

1 Department of Radiology, Severance Hospital, Yonsei University College of Medicine, 50 Yonsei-ro, Seodaemun-gu, Seoul 03722, South Korea

2 Department of Surgery, Yonsei University College of Medicine, 50 Yonsei-ro, Seodaemun-gu, Seoul 03722, South Korea

3 Department of Radiology, Memorial Sloan Kettering Cancer Center, New York, NY, USA

4 Department of Radiology, Research Institute of Radiological Science, Yonsei Biomedical Research Institute, 50 Yonsei-ro, Seodaemun-gu, Seoul 03722, South Korea 
Table 3 Distribution of LI-RADS category with CT and MRI

CT $(n=203)$

Pathologically viable $(n=120) \quad$ Pathologically nonviable $(n=83)$

MRI $(n=84)$

Pathologically viable $(n=58) \quad$ Pathologically nonviable $(n=26)$

LR-TR viable

Reader $1 \quad 47$ (39.2)

Reader 247 (39.2)

LR-TR equivocal

Reader $1 \quad 19$ (15.8)

Reader 217 (14.2)

LR-TR nonviable

Reader 154 (45.0)

Reader 256 (46.7)

\section{$5(6.0)$}

2 (2.4)

$2(2.4)$

$0(0.0)$

76 (91.6)

81 (97.6)
44 (75.9)

42 (72.4)

3 (3.6)

2 (3.4)

11 (19.0)

14 (24.1)
5 (19.2)

3 (11.5)

2 (7.7)

$2(7.7)$

19 (73.1)

$21(80.8)$

Data in parentheses are percentages. Percentages may not sum to $100 \%$ because of rounding off

Publisher's note Springer Nature remains neutral with regard to jurisdictional claims in published maps and institutional affiliations. 